\title{
Quantitative evidence of an intrinsic luminosity spread in the Orion nebula cluster
}

\author{
M. Reggiani ${ }^{1}$, M. Robberto ${ }^{2}$, N. Da Rio ${ }^{2}$, M. R. Meyer ${ }^{1}$, D. R. Soderblom ${ }^{2}$, and L. Ricci ${ }^{3}$ \\ 1 Institute of Astronomy, ETH Zurich, 8093 Zurich, Switzerland \\ e-mail: reggiani@phys. ethz.ch \\ 2 Space Telescope Science Institute, 3700 San Martin Drive, Baltimore, MD 21218, USA \\ 3 European Southern Observatory, Karl-Schwarzschild-Strasse 2, 85748 Garching, Germany \\ Received 23 March 2011 / Accepted 2 August 2011
}

\section{ABSTRACT}

\begin{abstract}
Aims. We study the distribution of stellar ages in the Orion nebula cluster (ONC) using accurate HST photometry taken from HST Treasury Program observations of the ONC utilizing the cluster distance estimated by Menten and collaborators. We investigate whether there is an intrinsic age spread in the region and whether the age depends on the spatial distribution.

Methods. We estimate the extinction and accretion luminosity towards each source by performing synthetic photometry on an empirical calibration of atmospheric models using the package Chorizos of Maiz-Apellaniz. The position of the sources in the HR-diagram is compared with different theoretical isochrones to estimate the mean cluster age and age dispersion. On the basis of Monte Carlo simulations, we quantify the amount of intrinsic age spread in the region, taking into account uncertainties in the distance, spectral type, extinction, unresolved binaries, accretion, and photometric variability.

Results. According to the evolutionary models of Siess and collaborators, the mean age of the Cluster is 2.2 Myr with a scatter of few Myr. With Monte Carlo simulations, we find that the observed age spread is inconsistent with that of a coeval stellar population, but in agreement with a star formation activity between 1.5 and 3.5 Myr. We also observe some evidence that ages depends on the spatial distribution.
\end{abstract}

Key words. stars: formation - stars: low-mass - stars: pre-main sequence - methods: statistical - techniques: photometric open clusters and associations: individual: Orion nebula cluster

\section{Introduction}

The analysis of the age distribution of pre-main sequence (PMS) stars in young clusters represents the most direct way of deducing how star formation proceeds in molecular clouds (Hartmann 2001; Hillenbrand et al. 2008; Da Rio et al. 2010b). Ages of young stellar objects are generally estimated by comparing the position of individual stars on the HR-diagram with theoretical isochrones and evolutionary tracks. For the majority of stars, the main uncertainties in age are related to the determination of the intrinsic luminosity, since the pre-main-sequence evolution of low mass stars takes place at nearly constant effective temperature. As pointed out by Hartmann (2001), the main sources of error in the luminosity are the uncertainties in the source distance, photometric variability, extinction, unresolved binary companions, and accretion. The discrepancies between different families of pre-main-sequence evolutionary models add further uncertainty, in particular the different slopes of the isochrone lines in the HR diagrams make it difficult to unequivocally derive not only the absolute but also the relative ages of cluster members.

Additional physical processes may affect the intrinsic luminosity of PMS stars. Baraffe et al. (2009) present calculations where episodic accretion at early stages of evolution of low-mass stars provides a possible explanation of the observed spread in the HR diagram. Hosokawa et al. (2011) show that, even though episodic accretion may occur, it is unable to explain the observed scatter. The spread obtained varying the initial radius or the thermal efficiency is much larger than the spread that results from different accretion histories, even though it is not enough to account for all the observed spread in the HR diagram (Hosokawa et al. 2011). However, on the one hand, a model quantifying whether the accretion-induced luminosity spread is able to account for the observations has not yet been created. On the other hand, we lack direct observational proof of this scenario. Hence, we still interpret the distribution of radii for a given mass as a spread in age.

In this paper, we revisit the age spread problem on the basis of new data from the Orion nebula cluster (ONC) obtained with the Hubble Space Telescope (HST), to complement the partial discussion of Da Rio et al. (2010a) based on ground based photometry. Our present goal is to assess the possibility of inferring robust age distributions using high precision multi-band photometry. Fitting all available photometric data simultaneously, we are able to accurate derive extinction and accretion luminosity, hence more tightly constrain the errors in the luminosity and the age of each source. We also focus on the assessment of apparent age spread induced by these errors. After a brief description of the photometry used and the selection criteria of our source sample (Sect. 2), we present the analysis carried out to assemble an observed HR diagram for the ONC (Sect. 3). The study of the relative uncertainties and the results obtained are described in Sects. 4 and 5. Finally, we discuss the possibility of a true age spread in the cluster (Sect. 6) and the dependence of age on the spatial distribution (Sect. 7).

\section{Photometry}

The data used for the present work consist mainly of broad-band photometry extracted from the large data-set of 
Table 1. ACS/WFC filters.

\begin{tabular}{lcc}
\hline \hline Filter & Ground equivalent & Exposure time (s) \\
\hline$F 435 W$ & Johnson $B$ & 420 \\
$F 555 W$ & Johnson $V$ & 385 \\
$F 658 N$ & $\mathrm{H} \alpha+[\mathrm{N} \mathrm{II}] \lambda 6583$ & 340 \\
$F 775 W$ & Cousin IC & 385 \\
$F 850 L P$ & z-Band & 385 \\
\hline
\end{tabular}

ACS/WFC observations collected for the HST Treasury Program on the Orion nebula cluster (GO-10246, PI M. Robberto). This program has covered an area of about $450 \mathrm{arcmin}^{2}$, roughly centered on the Trapezium Cluster in five filters. In Table 1, we list the ACS filters, their ground-based telescope equivalents, and the duration of individual exposures. The complete ACS/WFC catalog for the ONC includes 3200 sources (Robberto, in prep.) that have been classified as either a single star, binary star, photo-evaporated disk, dark silhouette, or possible background galaxy. A subset of the catalog describing the non stellar objects was presented by Ricci et al. (2008).

The deep ACS observations, designed to sample the substellar component of the cluster, do not provide photometry for the brightest sources because of saturation. We therefore complemented the ACS survey with ancillary ground-based data, collected within the same HST Treasury Program. These observations were obtained simultaneously at the MPG-ESO 2.2-m Telescope at La Silla with the Wide Field Imager (WFI) (Da Rio et al. 2009) and with the Infrared Side Port Imager (ISPI) at the CTIO Blanco 4-m Telescope (Robberto et al. 2010), fully covering the area imaged with the HST. In particular, for our analysis we selected the WFI data, which were taken in the $U$, $B, V$ and $I$ passbands, in a narrow-band filter centered on the $\mathrm{H}_{\alpha}$ line and in a medium resolution filter centered at $6200 \AA$. This provides a $R$-band line-free equivalent passband for stars $\mathrm{K}$-type and earlier, whereas for stars M-type and later it is centered on a temperature-sensitive $\mathrm{TiO}$ absorption band, which allows a reddening-independent measurement of $T_{\text {eff }}$ for cool stars (Da Rio et al. 2009). WFI measures were used only for stars not present in the ACS dataset. To avoid introducing color errors due to source variability, we did not combine the ACS and WFI data, as they were obtained at different epochs.

To reliably build the HR diagram, one has to combine the photometric observations with spectral types. From our original sample of 2793 objects, we have therefore selected 807 stars with spectral type given by Hillenbrand (1997), 100 stars with spectral type from a follow-up to the study of Stassun et al. (1999; see also Da Rio et al. 2010a), and 150 stars with spectral type from Da Rio et al. (2009), obtained with narrow-band photometry at $6200 \AA$ on a TiO absorption feature. In total, we therefore have 1057 stars with known spectral type and photometry in at least three bands at optical wavelengths. The completeness of our spectroscopic sample is analyzed in Sect. 5.1.

\section{HR diagram}

\subsection{Construction}

The construction of an accurate HR diagram requires the knowledge of several quantities and transformation relations. First, the spectral type has to be converted to effective temperature. For this purpose, we used the relation between spectral type and effective temperature given by Luhman (1999), which is tuned to bring the presumably coeval components of the young system
GG Tau onto the same isochrone, according to the Baraffe et al. (1998a) models. For M-type PMS stars, this relation is intermediate between dwarfs and giants and, as shown by Da Rio et al. (2009), it is more reliable than the relation assumed by Hillenbrand (1997). Using this transformation the temperatures of our sample range from $\sim 2500 \mathrm{~K}$ to $\sim 8000 \mathrm{~K}$. Second, to estimate the bolometric luminosity of each source one has to convert the observed magnitudes to absolute magnitudes adopting values for extinction, distance modulus, and bolometric corrections. One should also account for non-photospheric sources of luminosity, such as accretion luminosity.

For the ACS data, we used as a reference the F850LP filter ( $z$-band equivalent) using the relation

$\log \left(L_{\star} / L_{\odot}\right)=0.4\left[z_{\mathrm{ACS}, \odot}-\left(z_{\mathrm{ACS}, \star}-\Delta z_{\mathrm{acc}}-A_{z}+B C_{z, \star}(T)-D M\right)\right]$

where $z_{\mathrm{ACS}, \odot}$ is the absolute magnitude of the Sun, $z_{\mathrm{ACS}, \star}$ is the magnitude of the target star, $\Delta z_{\text {acc }}$ is the estimated contribution to the observed brightness due to disk accretion, $A_{z}$ is the extinction, $B C_{z, \star}$ is the bolometric correction, and $D M$ is the distance modulus. For the WFI data, we used an analogous expression for the $I$ band.

To estimate the various terms, we computed synthetic photometry for families of stellar atmospheric models using the SED fitting package Chorizos (Maíz-Apellániz 2004). The $\chi^{2}$-minimization SED-fitting enables us to assess the correspondence between observed and synthetic photometry up to $N=4$ physical parameters. In other words, Chorizos solves the problem of finding which model SED is the most compatible with the observed colors in a model $\mathrm{N}$-dimensional parameter space. We assembled the spectral libraries of various authors: Kurucz (1993) for effective temperatures larger than $8000 \mathrm{~K}$, NextGen model atmospheres (Hauschildt et al. 1999) for $5000 \leq T \leq$ $8000 \mathrm{~K}$, and AMES-MT 2000 models (Allard et al. 2000) for $T \leq 5000$, all for solar metallicity in accordance with Da Rio et al. (2010a).

Da Rio et al. (2010a) show that the colors of ONC members differ from those of main-sequence dwarfs in the M-type regime and that the atmospheric models do not accurately match the photometry of the ONC. Therefore, we limited the parameter space to models corresponding to the empirical calibration suggested by Da Rio et al. (2010a) as a good approximation for the ONC. This empirical isochrone was built to obtain a good match for a selected sample of non-accreting low-extinction members of the ONC, using the ZAMS intrinsic colors for $T \geq 3700 \mathrm{~K}$, the 2 Myr synthetic colors for $T \leq 2900 \mathrm{~K}$, and a linear interpolation between the two for $2900 \geq T \leq 3700 \mathrm{~K}$.

To account for the accretion luminosity that may affect our young sources, we added a typical accretion spectrum to the family of template spectra. According to Calvet \& Gullbring (1998), the total accretion column emission can be well approximated by the superposition of an optically thick emission from the heated photosphere below the shock with an optically thin emission generated in the infalling flow, in a 3:1 ratio. The optically thick component can be modeled by a black body at $T_{\text {eff }}=6000-8000 \mathrm{~K}$, and we assumed $T_{\text {eff }}=7000 \mathrm{~K}$. For the optically thin emission, we used Cloudy (Ferland et al. 1998) to generate the spectrum of an optically thin slab with $n=10^{8} \mathrm{~cm}^{-3}$ also at $T \simeq 7000 \mathrm{~K}$. We added the combined spectrum to our reference stellar models with ratios of $\log \left(L_{\mathrm{accr}} / L_{\mathrm{tot}}\right)$ increasing from -3 to 0 .

We ran Chorizos for each source of our sample, assuming the metallicity and the stellar effective temperature as fixed parameters and two different reddening laws, corresponding to the standard $R_{V}=3.1$ and $R_{V}=5.5$, which is considered by some 

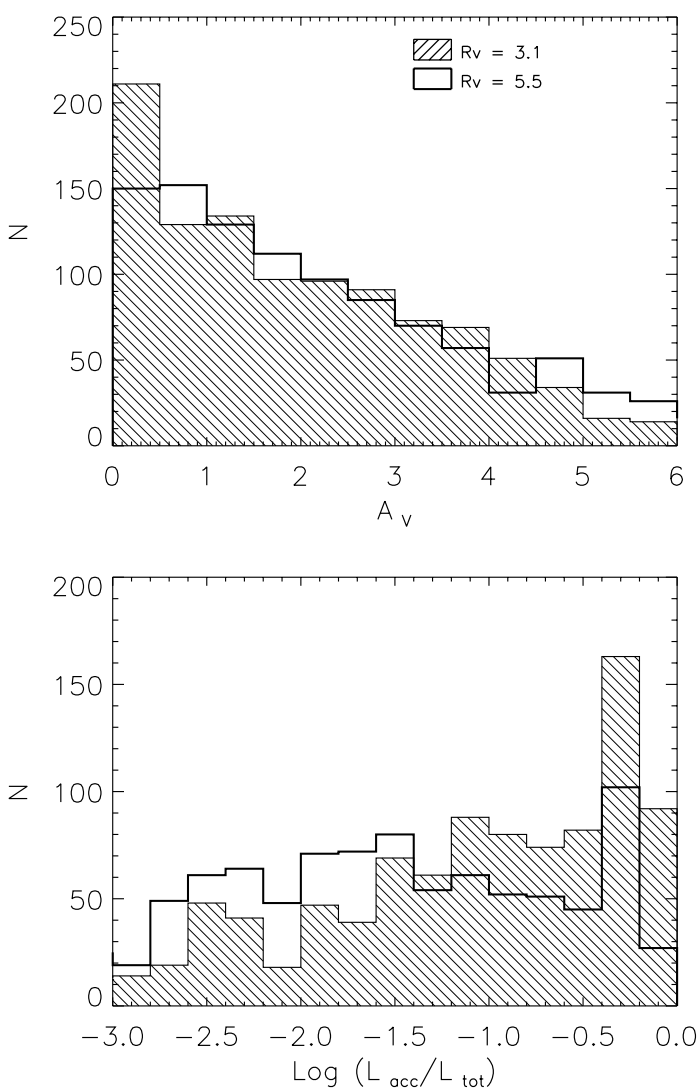

Fig. 1. Extinction and accretion luminosity. Top: distribution of the extinction for the sample of 1057 stars from the ACS and WFI catalogs for which we have estimated extinction and accretion luminosity. Bottom: distribution of the accretion luminosity for the same sample of objects. The accretion luminosity is expressed in terms of $L_{\text {accr }} / L_{\text {tot }}$ and in a logarithmic scale ranges from -3 to -0 .

authors to be more appropriate for the Orion nebula (Johnson 1967; Costero \& Peimbert 1970). Therefore, extinction and accretion represent the variables of our fitting procedure. Chorizos returned the most likely value of extinction and accretion luminosity for each source, for each reddening law. With Chorizos, we can fit all available photometric data at the same time to derive extinction and accretion luminosity for each source, whereas Da Rio et al. (2010a) concentrated on two colors. Chorizos is therefore less sensitive to the effects of the occasional spurious data points, or the data with the highest photometric error. In Fig. 1, we show the distributions of the extinction and accretion luminosity for the two choices of reddening law. For the standard reddening law, that we adopt as our main reference, the extinction distribution has a median value at $A_{V}=2.2 \mathrm{mag}$ and shows that roughly $70 \%$ of the sources are extincted by less than $3 \mathrm{mag}$. From the distribution of $\left(L_{\mathrm{accr}} / L_{\mathrm{tot}}\right)$, we infer an average value for the relative fraction of $5 \%$ and that more than half of the stars in the sample display an excess that can be attributed to accretion with $L_{\mathrm{accr}} / L_{\mathrm{tot}}$ greater than $3 \%$ (comparable to what was found by Da Rio et al. 2010a).

The bolometric corrections appropriate for our population were derived directly from the integration of the synthetic spectra in $z$, as well as across all wavelengths:

$B C(T)=-2.5 \log \left[\frac{\int_{\lambda} S_{\lambda} F_{\lambda}(T) \mathrm{d} \lambda}{\int_{\lambda} F_{\lambda}(T) \mathrm{d} \lambda} / \frac{\int_{\lambda} S_{\lambda} F_{\lambda}(\odot) \mathrm{d} \lambda}{\int_{\lambda} F_{\lambda}(\odot) \mathrm{d} \lambda}\right]$, where $F_{\lambda}(T)$ is the spectral energy distribution of the star of temperature $T$, while $F_{\lambda}(\odot)$ is the solar spectral profile and $S_{\lambda}$ is the passband of the filter multiplied by the quantum efficiency function of the detector. According to Eq. (1), the bolometric correction of the Sun is zero in each band, whereas the zero-points in this case are irrelevant as they cancel out in the calculation of the luminosity.

We also integrated the fluxes of a NextGen synthetic solar spectrum, assuming the canonical value of $M_{V, \odot}(V$ Johnson $)=$ +4.83 (Binney \& Merrifield 1998). The absolute magnitudes of the Sun in the $z$ and $I$ bands of ACS and WFI are found to be, respectively, $M_{z}=4.002$ and $M_{I}=4.020$, computed with synthetic photometry for the solar spectrum. We assumed the distance value of $d=414 \pm 7$ pc measured by Menten et al. (2007), which corresponds to a distance modulus of $D M=8.085$.

\subsection{Results}

After estimating the temperature and luminosity of each source, we produced the HR diagrams for the two values of reddening laws mentioned above. We then compared the position of the stars with the three sets of evolutionary tracks and isochrones of Siess et al. (2000), Palla \& Stahler (1999), and D'Antona \& Mazzitelli (1997) (in its extended form of 1998).

In Fig. 2, we show the HR diagram with the reddening law $R_{V}=3.1$ and the calculations of Siess et al. (2000). There is currently no family of evolutionary models showing a significantly higher consistency with dynamical mass estimates (Hillenbrand \& White 2004). Therefore we adopt the calculations of Siess et al. (2000) as our reference, as they provide the smallest apparent age spread in the HR diagram. According to Da Rio et al. (2010a), these models reduce the systematic correlation of average age with mass, which is more prominent when using either the isochrones of Palla \& Stahler (1999), or D'Antona \& Mazzitelli (1994) models (Hillenbrand 1997). In Fig. 2, the objects are indicated according to their dataset and the available photometry, together with evolutionary tracks and isochrones. In the next section, we analyze the accuracy of our estimate of the luminosity to quantify how much of the observed scatter can be produced by observational uncertainties. Then, in Sect. 5, we explicitly derive the age of each star by means of interpolation over the tracks and the isochrones to study the age distribution of our sample and quantify the amount of scatter in terms of age spread.

\section{Sources of uncertainties}

As mentioned before, there are concerns about the reliability of ages, as well as masses, derived from the HR diagram of young clusters, owing both to the discrepancies between different PMS evolutionary models and to the various uncertainties affecting observations. Although identifying the optimal model goes beyond the scope of this work, we can take a fresh look at the observational errors listed by Hartmann (2001) and discuss how they may affect our results on the ONC. First of all, we present the analytical estimate of the uncertainties in luminosity and age, whereas in Sect. 4.2 we describe a Monte Carlo approach to the estimate of the same quantities.

\subsection{Analytical estimates}

Distance. The distance obtained with VLBI (Very Long Baseline Array) by measuring the trigonometric parallax of radio sources 


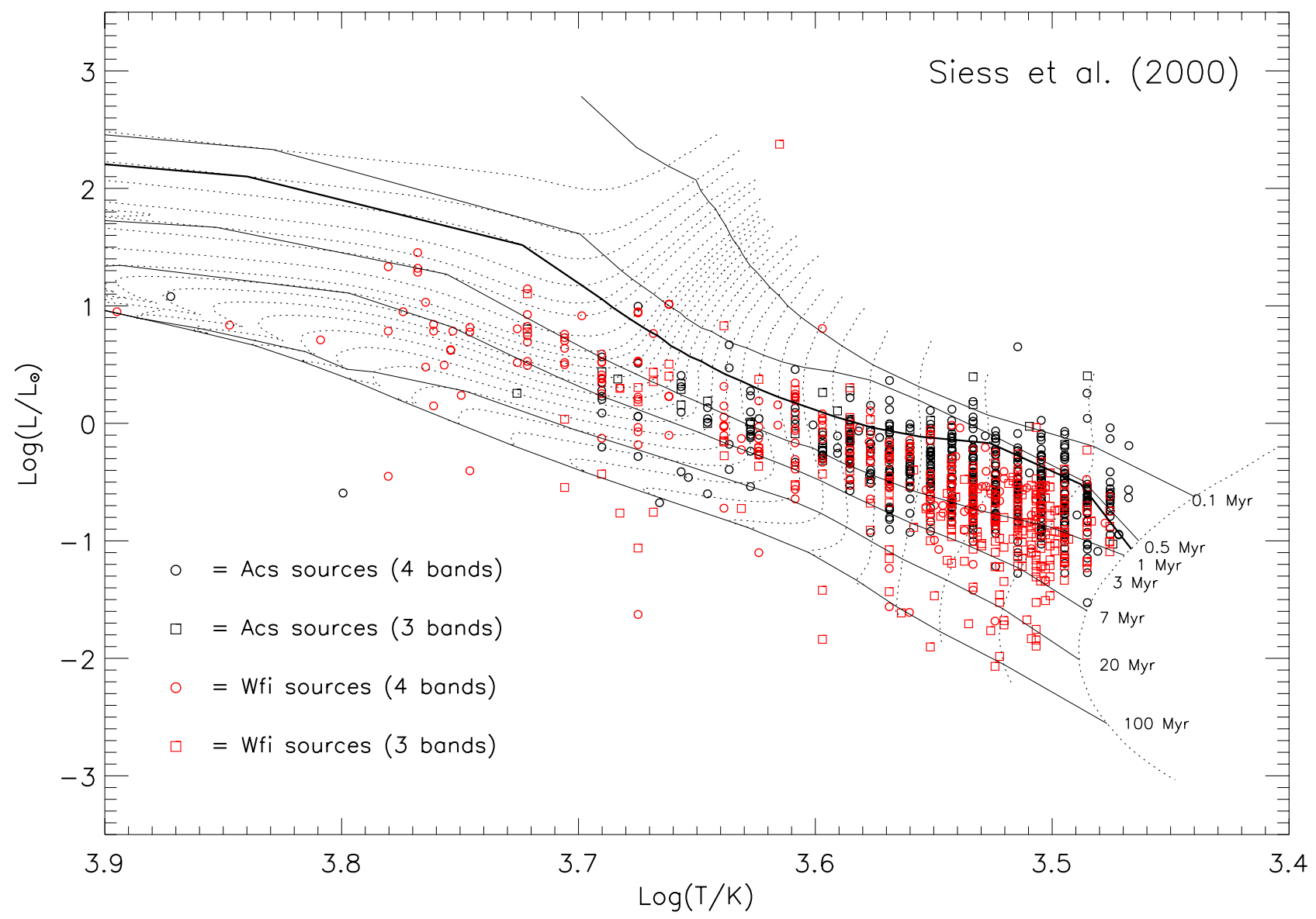

Fig. 2. HR diagrams for the ONC. Black symbols represent ACS sources: circles are the stars with four-bands photometry while squares represent stars with complete three-bands photometry. Red squares indicate respectively WFI objects with four-band photometry, while red diamonds the WFI stars with three-bands photometry. Superimposed are the evolutionary calculations of Siess et al. (2000). Solid lines represent isochrones, the thickest one indicating the $1 \mathrm{Myr}$ isochrone. Dotted lines represent evolutionary tracks and they range from $0.1 \mathrm{up}$ to $5 \mathrm{M}_{\odot}$.

in the $\mathrm{ONC}$ gives $d=414 \pm 7 \mathrm{pc}$. The standard deviation is larger than the transversal size (about $3 \mathrm{pc}$ ) of the cluster, hence larger than the intrinsic spread in distances within the cluster. We can therefore assume that $\sigma(\log L)=0.015$.

Spectral type. The spectral types given by Hillenbrand (1997) are accurate to within one sub-type. According to Hartmann (2001), errors in the spectral type tend to cancel; for instance a star that is erroneously assigned an earlier spectral type would have a larger age at a constant luminosity, but the extinction would also be overestimated and consequently the luminosity erroneously higher. Averaging over the spectral type range of K6-M1, Hartmann (2001) found that an error within a subtype in spectral type corresponds to an error in age of $\sigma(\log t)=0.02$. Da Rio et al. (2010a) demonstrated that even when varying the effective temperature within its error and consequently changing the bolometric correction to calculate the luminosity, owing to variations in extinction, the error in the luminosity remains parallel to the isochrone (see e.g. Fig. 17 in Da Rio et al. 2010a).

Extinction. Chorizos provide a typical uncertainty in the extinction of $\sigma\left(A_{V}\right) \sim 0.2 \mathrm{mag}$. Using Eq. (1) to propagate errors, and knowing that $A_{I} \sim 0.5 A_{V}$ and $A_{z} \sim 0.4 A_{V}$, this translates into $\sigma(\log L) \sim 0.05$.

Photometric variability. According to Herbst et al. (2002), the average variation in magnitude owing to photometric variability for the ONC is $\Delta I=0.18 \mathrm{mag}$. Following the same authors, we use the standard deviation to characterize the level of variability, since a general peak-to-peak measure can be affected by single accidental error. This standard deviation (0.075 mag) gives a logarithmic uncertainty on luminosity of $\sigma(\log L)=0.03$.

Unresolved binary companions. With a pixel scale of $50 \mathrm{~ms}$, $\mathrm{ACS}$ is able to resolve sources of comparable brightness with separations larger than $\sim 50 \mathrm{AU}$ at the distance of the cluster. Assuming the standard lognormal period/separation distribution that peaks at approximately 30 AU (Duquennoy \& Mayor 1991) and a binary fraction similar to the one observed in the field (Reipurth et al. 2007), we have a probability that $20 \%$ of all sources have unresolved binary companions. With this probability, the basic model described in Hartmann (2001) allows us to derive a logarithmic error in luminosity caused by unresolved binaries of $\sigma(\log L)=0.016$.

Accretion. Our analysis then allows us to derive and correct for the amount of accretion luminosity that affects each source. However, the error in $L_{\text {acc }}$ is given by Chorizos in terms of $\sigma\left(\log \left(L_{\mathrm{accr}} / L_{\mathrm{tot}}\right)\right)$, and consequently depends on $\sigma\left(\log L_{\mathrm{tot}}\right)$, which is what we wish to determine. The optimal way to estimate the effect of this uncertainty in the luminosity is the use of Monte Carlo simulations. Therefore, we neglect this contribution in this preliminary calculation.

The quadratic sum of all these errors gives an overall uncertainty $\sigma(\log L)=0.06$, which is roughly one third of the value $\sigma(\log L)=0.16$ reported by Hartmann (2001) for the Taurus cloud. This reflects the higher quality of our data set. 
Table 2. Mean age and age dispersion.

\begin{tabular}{ccccc}
\hline \hline & \multicolumn{2}{c}{$R_{V}=3.1$} & \multicolumn{2}{c}{$R_{V}=5.5$} \\
PMS model & $\langle\log ($ age $)\rangle$ & $\sigma \log ($ age $)$ & $\langle\log ($ age $)\rangle$ & $\sigma \log ($ age $)$ \\
\hline Siess et al. (2000) & 6.36 & 0.38 & 6.30 & 0.46 \\
D'Antona \& Mazzitelli (1998) & 5.93 & 0.59 & 5.82 & 0.71 \\
Palla \& Stahler (1999) & 6.14 & 0.49 & 6.07 & 0.53 \\
\hline
\end{tabular}

It is known that the ages of low-mass pre-main-sequence stars on the Hayashi tracks exhibit an approximate power-law relation to the stellar luminosity $L$ for each mass, of the form $t \propto L^{-3 / 2}$ (Hartmann 2009). Owing to this relationship between luminosity and age, the error distribution in age takes the form $\delta t / t \sim \delta \log t$, with

$\sigma(\log t)=(3 / 2) \sigma(\log L)$

Therefore, from the uncertainty in the luminosity one can derive the error in the age, which turns out to be $\sigma(\log t)=0.09$. As also shown by Hartmann (2001), errors of this order may preserve a time dependence of the star formation rate possibly present in the observations, a dependence that is instead lost when the observational errors are $\sigma(\log t) \gtrsim 0.2$.

Since our preliminary estimate of the overall uncertainty turns out to be smaller than the limit indicated by Hartmann, we can be fairly confident that, from the observational point of view, our uncertainties may allow us to infer real age trends from the analysis of the HR diagram. However, to understand the extent to which this can be done, a more complete analysis is needed (see Sect. 6).

\subsection{Monte Carlo simulations.}

After roughly estimating the uncertainty in luminosity, we quantify the error in our age estimates more robustly by performing Monte Carlo simulations to evaluate the dispersion in the estimated absolute luminosity of each object caused by all the sources of uncertainty indicated in Sect. 4.1. By generating artificial errors for each star, we can more accurately determine the overall uncertainties in the bolometric luminosity.

To evaluate the extinction and the accretion luminosity, we executed Chorizos varying the photometry according to the photometric errors and adding variability, following the variability distribution in the $I$ band given by Herbst et al. (2002). We computed the amount of variation in the other bands using synthetic photometry of artificial stars with standard spot temperatures, radii, and filling factors for $\mathrm{T}$ Tauri stars (Berdyugina 2005). During each iteration, we also varied the value of the distance within its error and added a shift in luminosity due to binarity, assuming the probability of unresolved companions given in Sect. 4.

We ran $N=200$ trials for each source and determined the dispersion in our estimates of the luminosity, which we then equated to $\sigma(\log L)$. We averaged our results for the whole sample and assumed that the value of $\sigma(\log L)=0.10$ is representative of our mean uncertainty in the luminosity.

According again to Eq. (2), this error translates into an uncertainty in the estimate of the ages of $\sigma(\log t)=0.15$, which is larger than we derived from basic error propagation $(\sigma(\log t)=$ 0.09). A larger error was expected, because we also considered the uncertainty in accretion luminosity, which had not been taken into account in the previous calculation. Nevertheless, this value is below the $\sigma(\log t)=0.2$ threshold given by Hartmann (2001),

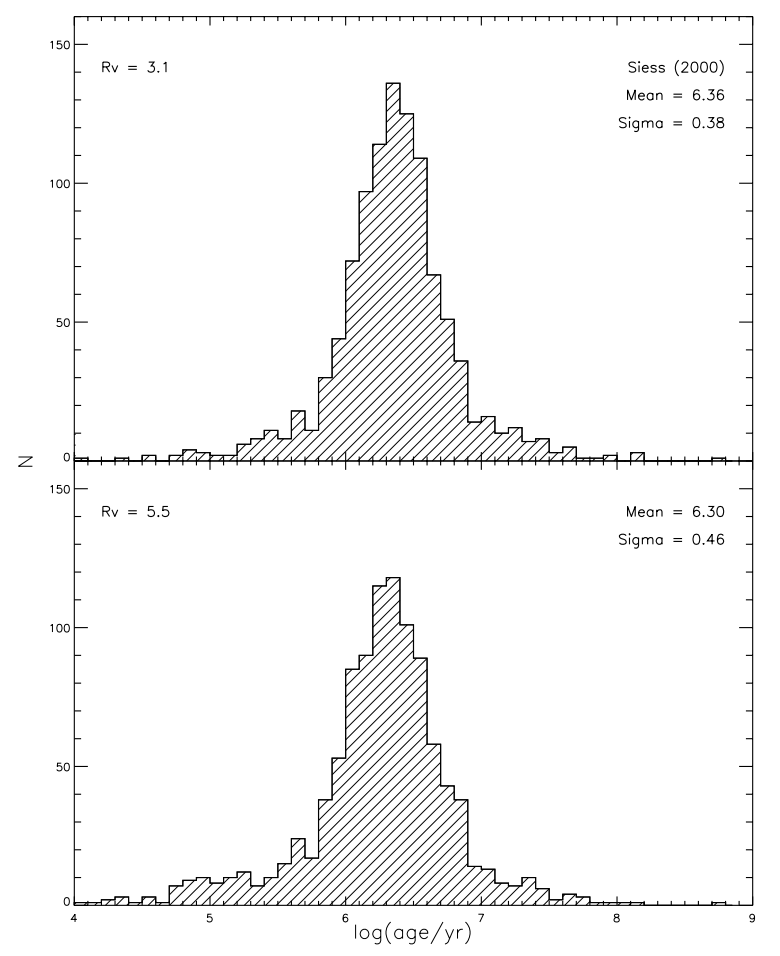

Fig. 3. Distributions of stellar ages. Histogram of the number of stars for a given age bin, as derived from Siess et al. (2000) evolutionary calculations for the reddening laws $R_{V}=3.1$ (top) and $R_{V}=5.5$ (bottom).

and clearly remains much smaller than the observed scatter (see Sect. 5).

\section{The age distribution}

In Fig. 3, we show the logarithmic distribution of stellar ages obtained for the two reddening laws we adopted and the Siess et al. (2000) evolutionary models. The average ages and age dispersions for the ONC that we derived with this set of evolutionary tracks and for the different reddening laws are listed in Table 2.

The cluster age turns out to depend on the reddening law. In general, ages derived assuming a reddening law of $R_{V}=3.1$ are older than those derived assuming $R_{V}=5.5$. This is expected, because high values of $R_{V}$ imply higher extinction values, hence higher intrinsic stellar luminosities and younger ages. The mean age predicted by Siess et al. (2000) for the sample is $\sim 2.3 \mathrm{Myr}$ and $\sim 2$ Myr for $R_{V}=3.1$ and $R_{V}=5.5$, respectively.

Using the information regarding variability supplied by Herbst et al. (2002), it is possible to select a sub-sample of 508 objects showing a peak-to-peak variability $\Delta I \leq 0.4$ and 298 objects with $\Delta I \leq 0.1$. For these two samples, the total error in the logarithm of the luminosity, as estimated by means of basic error propagation, goes to $\sigma(\log L)=0.053$ and $\sigma(\log L)=$ 0.051 , respectively (for the complete sample $\sigma(\log L)=0.06$, see Sect. 4). In Fig. 4, we show the distributions of the ages for 
Table 3. Selected samples: mean age and age dispersion (Siess et al. 2000).

\begin{tabular}{|c|c|c|c|c|c|c|}
\hline \multirow[b]{2}{*}{$R_{V}$} & \multicolumn{2}{|c|}{ Herbst Sample } & \multicolumn{2}{|c|}{ First selection } & \multicolumn{2}{|c|}{ Second selection } \\
\hline & $\langle\log ($ age $)\rangle$ & $\overline{\sigma \log (a g e)}$ & $\langle\log ($ age $)\rangle$ & $\overline{\sigma \log (a g e)}$ & $\langle\log ($ age $)\rangle$ & $\overline{\sigma \log (a g e)}$ \\
\hline 3.1 & 6.33 & 0.36 & 6.31 & 0.34 & 6.31 & 0.36 \\
\hline 5.5 & 6.37 & 0.43 & 6.25 & 0.42 & 6.27 & 0.41 \\
\hline
\end{tabular}

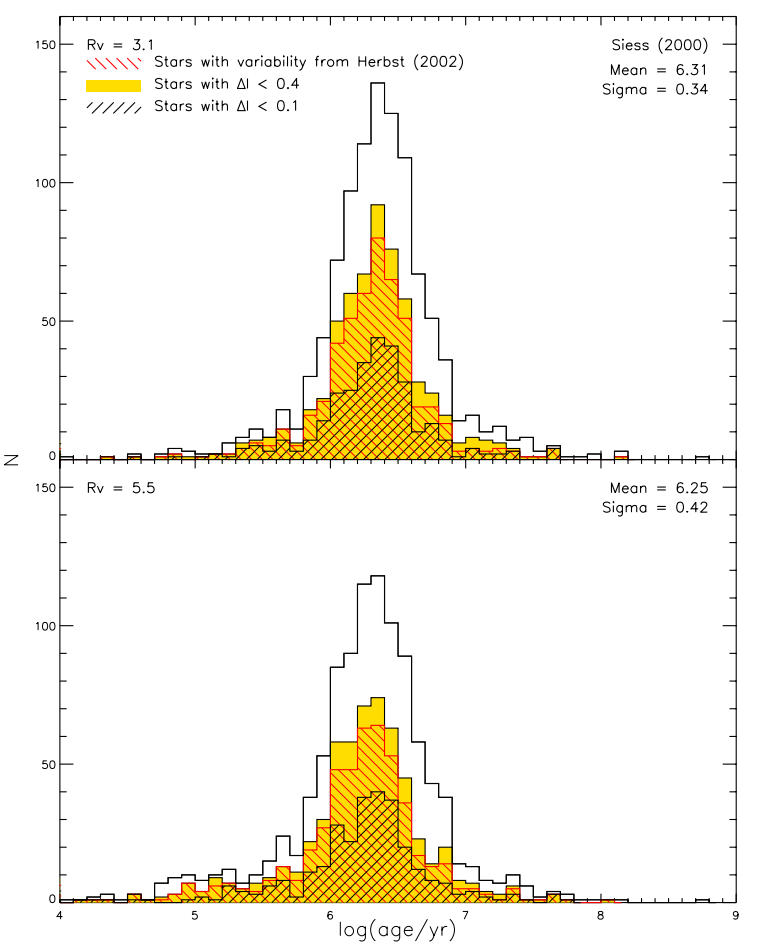

Fig. 4. Distributions of stellar ages for low variability stars. The stellar ages are derived from Siess et al. (2000) evolutionary calculations for the reddening laws $R_{V}=3.1$ (top) and $R_{V}=5.5$ (bottom). The open histogram represents the total sample. The yellow area indicates the sample of 608 stars for which we have information about variability from Herbst et al. (2002). The red hatched histogram represents the sources selected with $\Delta I \leq 0.4 \mathrm{mag}$, while the black one corresponds to stars with $\Delta I \leq 0.1$. The average age of the cluster and the age spread are relative to the third sample.

the different samples and in Table 3 we summarize the results. A direct comparison with the estimates obtained for the full sample does not show substantial differences in both the average age and age spread. The Kolmogorov-Smirnov test (KS test), which returns the probability that two distributions were drawn from the same parent sample, confirms that the samples are consistent with the original population, i.e. variability does not significantly affect the mean and the spread of the age distribution.

As mentioned before, we considered the three different families of PMS evolutionary tracks and isochrones of D'Antona \& Mazzitelli (1997), Siess et al. (2000), and Palla \& Stahler (1999). In Figs. 5 and 6, we present the HR diagram and the age distribution according to these different sets and in Table 2 the mean ages and the age spreads predicted for the ONC. The differences in the estimates are clearly visible. First of all, this discrepancy affects both the mean age of the cluster and the age of the single star. Regardless of the value of the reddening parameter, D'Antona \& Mazzitelli (1997) consistently predicts younger ages, which appear to be consistent with the results obtained for the same evolutionary calculations by Hillenbrand (1997). Second, the value of the mean age also depends on

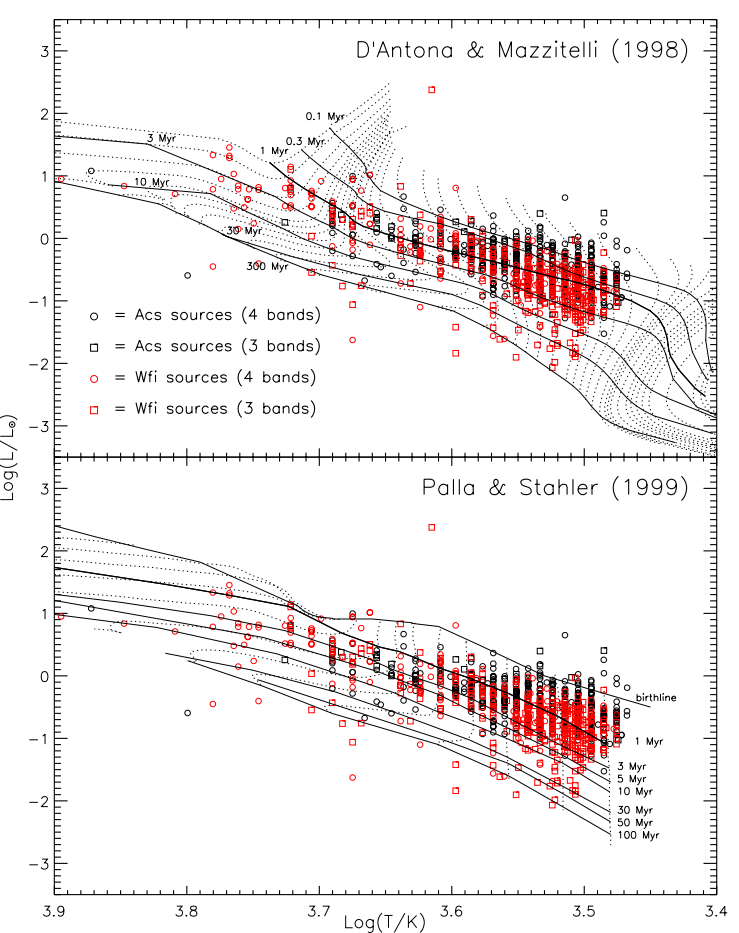

Fig. 5. HR diagrams for the ONC. Same legend as in Fig. 2. From the top to the bottom are superimposed evolutionary tracks and isochrone from D'Antona \& Mazzitelli (1997) and Palla \& Stahler (1999). Dotted lines represent evolutionary tracks and range from 0.017 to $3 M_{\odot}$ for D’Antona \& Mazzitelli and from 0.1 to $6 M_{\odot}$ for Palla \& Stahler.

the predictive capability of the model for this particular cluster. Since the different sets cover slightly different areas in the HR diagram (Fig. 5), certain stars may or may not be left out of certain models. Finally, a relative age spread appears to be robust regardless of which family of models is used, although the amount of this spread is model dependent. The scatter predicted by Siess et al. (2000) is the smallest one with both the standard and the anomalous reddening law.

In conclusion, from the observational point of view our sample enables us to place the stars in the HR diagram with fairly high precision, but different PMS evolutionary models provide significantly different ages. Nevertheless, regardless of the choice of the set of models, there appears to be an age scatter that cannot be accounted for by observational errors. Star formation in the ONC has mainly occurred within the past $10 \mathrm{Myr}$, with $\sim 70 \%$ of the stars being younger than 3 Myr. There is no evidence of a single star-formation burst as the distribution spans a wide range of ages with a scatter of a few Myr, in agreement with what was found by Hillenbrand (1997) and Da Rio et al. (2010a).

\subsection{Completeness analysis}

The HRD and the age distribution presented here, are relative to the subsample of ONC objects with at least three-band 


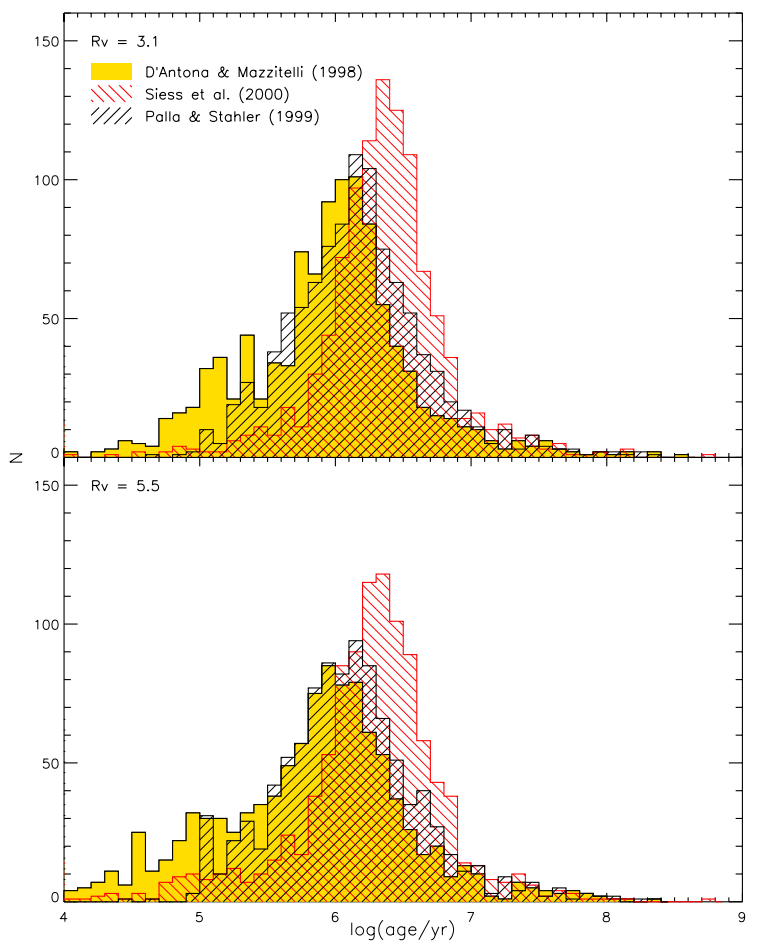

Fig. 6. Distributions of stellar ages for different PMS evolutionary models. These histograms correspond to the reddening laws $R_{V}=3.1$ (top) and $R_{V}=5.5$ (bottom). The yellow histogram represents the results obtained by interpolating the D'Antona \& Mazzitelli (1997) isochrones. The red hatched histogram corresponds to Siess et al. (2000), while the black hatched one represents Palla \& Stahler (1999) predictions.

photometry and known spectral types. This represents only a fraction of our entire photometric catalog of the $\mathrm{ONC}$, and its completeness, which worsen towards fainter luminosities - or lower masses - basically depends on the availability of spectral types, and not on the photometric detection. This is because, whereas our HST photometry extends well into the BD mass range and goes five magnitudes fainter than Hillenbrand (1997), spectral types reach at most the lowest masses in the stellar regime.

Towards this limit, the subsample also becomes extinction limited, in the sense that very low mass members have an available $T_{\text {eff }}$ estimate only if their $A_{V}$ is not significantly large. This is caused by the source of our spectral types, the majority of which are from the optical spectroscopy of Hillenbrand (1997), whose spectra were obtained by randomly selecting the candidates, but only for sources with previously available $V$ and $I$ photometry, and were clearly limited to lower magnitude limits. Other spectral types were derived by Da Rio et al. (2009) using a photometric index computed from ground-based measurements in the $V$ and $I$ bands, plus a medium-band filter centered on the TiO feature at $\lambda \sim 6200 \AA$; this second set reaches spectral types as late as M6, for moderate extinction.

Besides the optical luminosity constraint, no additional criterion was imposed (in both Hillenbrand 1997; and Da Rio et al. 2009) when selecting sources with assigned spectral types. Consequently, the only selection effect affecting the completeness of our subsample is the apparent $V$ magnitude of the members, since owing to the intrinsic color and reddening of typical ONC populations the detection is generally easier at longer wavelengths.
We therefore computed the completeness distribution of our subsample as a function of $V$ magnitude. Even at the faintest $V$ magnitudes, the ACS photometric catalog can be considered complete, reaching several magnitudes deeper than Hillenbrand (1997). Hence, the ratio of the two $V$-band luminosity functions (of the subsample to the whole ACS catalog) automatically provides the $V$-band completeness function. More precisely, this computation was done separately in three subcases: the ACS stars, the WFI stars in the FOV of ACS, and the WFI stars outside the ACS FOV. Since at the bright end of the LF, the ACS catalog is deficient of sources detected instead with WFI, owing to saturation, we complemented the ACS catalog with the missing bright WFI sources falling in the same area. By adding WFI sources outside the ACS FOV, we permitted the completeness function to be $>100 \%$ for some bright $V$-magnitudes; however, this is not a problem, since any multiplicative renormalization of the completeness function is absolutely arbitrary and irrelevant to our purposes.

We now move from the $V$-band completeness to the completeness as a function of stellar parameters, such as $T_{\text {eff }}$ and $\log L$, or more precisely, mass and age. This cannot be done directly, because differential reddening, as we have said, introduces an extinction-limited bias. The computation is still possible in a probabilistic way using a Monte Carlo simulation. The method is identical to that used in Da Rio et al. (2010a): we consider a pair of values of mass and age. Assuming the evolutionary models, we convert this point into $T_{\text {eff }}$ and $\log L$. Then we apply backwards bolometric corrections and intrinsic colors to convert this point to the $V$ vs. $V-I \mathrm{CMD}$, assuming $A_{V}=0$. Having the intrinsic $V_{0}$ magnitude of the (mass, age) point, we redden this test star randomly, with values of $A_{V}$ drawn from the reddening distribution of the $\mathrm{ONC}$, computed on the brightest half of the population. Each of this "reddened" test stars have a different (fainter) $V$ magnitude than $V_{0}$, hence a different completeness. By simply averaging all these completeness ratios, we derive the exact completeness of the point in the mass-age plane considered at first. Iterating this procedure for each (mass, age) pair, we derive a two-dimensional completeness function in the mass-age plane.

Figure 7 shows the completeness contours in the mass-age plane for the two different reddening laws, whereas in Fig. 8 we present the completeness-corrected age distributions of the Siess et al. (2000) evolutionary models for the two different reddening laws. We observe that completeness decreases toward lower masses and older ages, therefore the completeness correction does not significantly change the average ages and the measured age spread $(\langle\log ($ age $)\rangle=6.40$ and $\sigma \log ($ age $)=0.49$ for $\left.R_{V}=3.1\right)$ but extends the old age end of the distributions to older ages.

However, optical photometry alone is often insufficient to detect all of the stars in young clusters. Near-infrared surveys of the ONC in the past few decades have revealed large numbers of highly extincted sources in this region (e.g. Hillenbrand \& Hartmann 1998; Muench et al. 2002), even though a recent near-infrared study has shown that the majority of stars $(\sim 70 \%)$ of the ONC have $A_{V}<5$ (Robberto et al. 2010; Scandariato et al. in prep.). Moreover, the contamination becomes significant as the sensitivities of the surveys increase. Hence, it is incorrect to assume that everything that has been observed in the nearinfrared is a cluster member. In any case, because our survey is not complete down to $0.25 M_{\odot}$ for more deeply embedded association members, we tested whether our sample is biased toward older stars. We considered a mass-age- $A_{V}$ limited sample $\left(M<0.25 M_{\odot}, t<10^{7} \mathrm{Myr}\right.$ and $\left.A_{V}<5\right)$ of 647 stars 

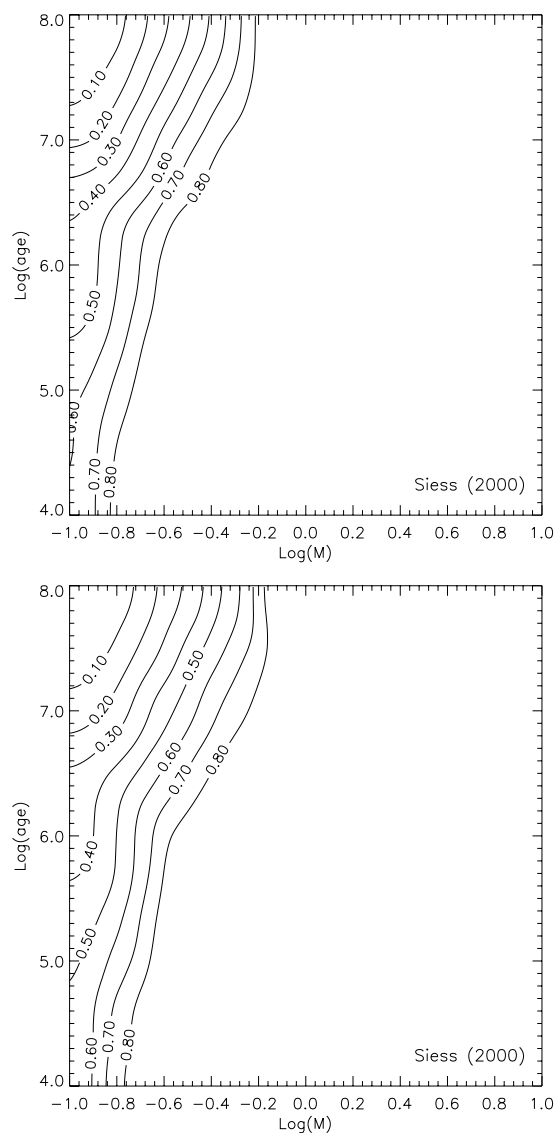

Fig. 7. Contour plots of the completeness function in the mass/age plane, computed from our simulations for the evolutionary models of Siess et al. (2000) and for $R_{V}=3.1$ (top) and $R_{V}=5.5$ (bottom).

in total. We divided this subsample into two halves with equal numbers of objects of low $\left(A_{V} \lesssim 2\right)$ and high $\left(A_{V} \gtrsim 2\right)$ extinction. We used the KS test to check whether the age distributions of the two samples are drawn from the same parent population. We are unable to reject the hypothesis that the distributions are the same $\left(P(d)_{\mathrm{KS}}=0.76\right)$. Since we do not find any evidence that the age distribution changes with increasing $A_{V}$, we argue that since we included most of the stars, though not all, in our sample we can study the age distribution of the cluster based on a lower extinction sub-sample.

\subsection{Membership}

It could be argued that some of the scatter observed in the HR diagram and the age distribution might be due to non-member contamination. The Orion complex itself consists of three subgroups, the ONC (Orion subgroup Id) and another three subgroups of stars (Ia, Ib, and Ic; Brown et al. 1994). The most likely subgroup from which we would see contamination in our data is the Orion subgroup Ic, since it is located along the same line of sight to the ONC. Orion Ic is thought to be less than $5 \mathrm{Myr}$ old according to Brown et al. (1994), thus we would expect few differences between the isochrones of the two subgroups.

For the ONC objects also present in H97, we utilized the membership given in that work, which had been taken from the proper motion study of Jones \& Walker (1988), to determine the age distribution of the confirmed members. Among our 1057 stars, 701 have proper motion measurements and are thought to be ONC members with a probability $P>50 \%$. In

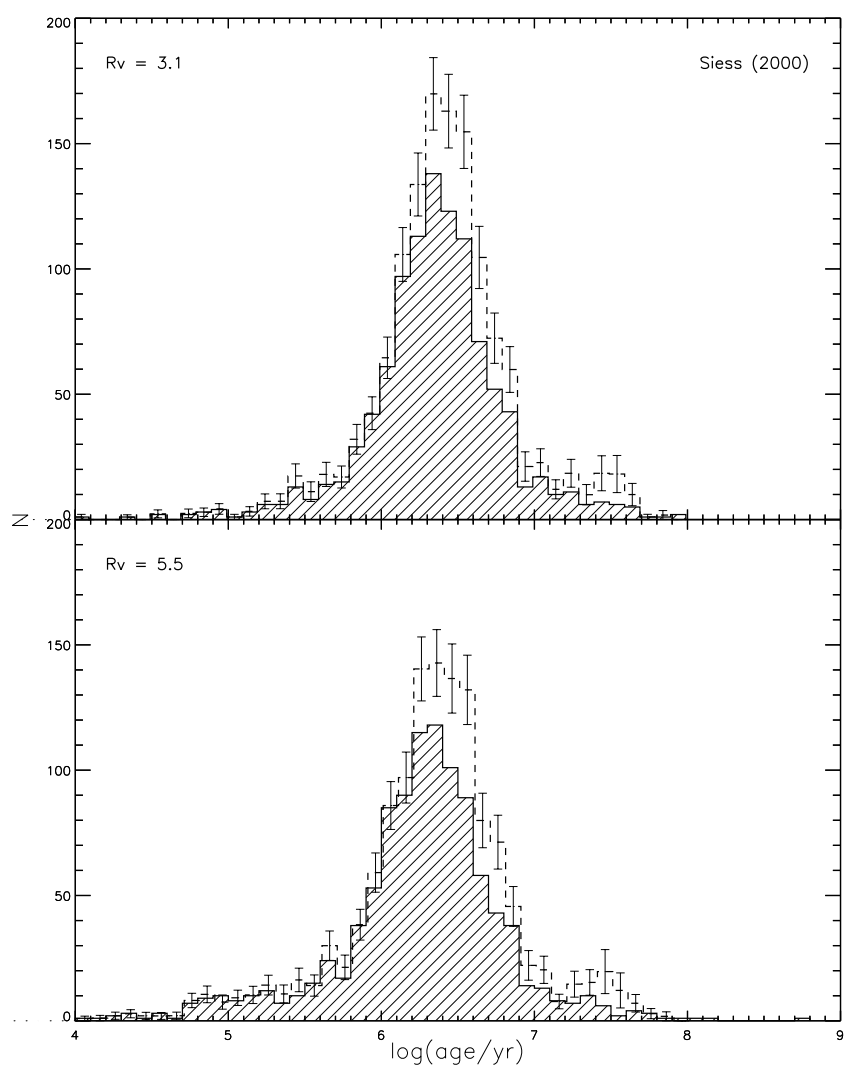

Fig. 8. Completeness-corrected age distribution. The hatched histogram represents the age distribution of our sample. The open histogram shows the age distribution of the completeness-corrected sample.

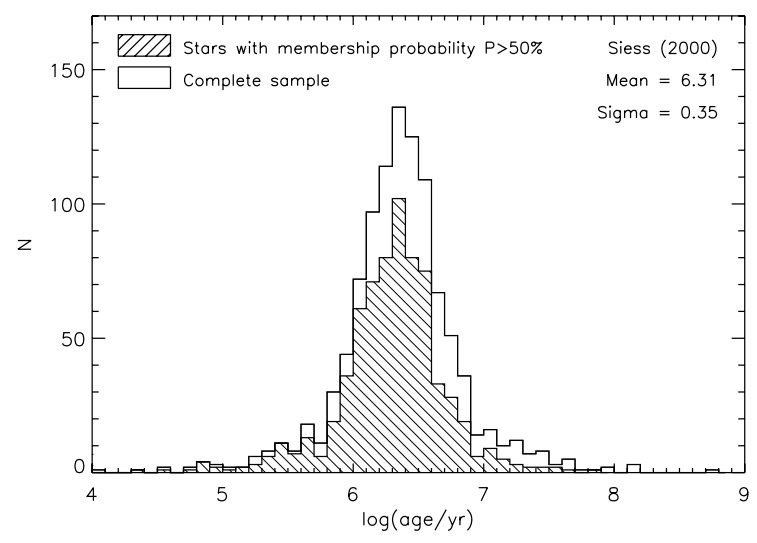

Fig. 9. Age distribution of confirmed members. The open histogram represents the age distribution of the complete sample. The hatched histogram shows the age distribution of the stars also present in the H97 catalog with membership probability $P>50 \%$ according to the proper motion survey of Jones \& Walker (1988). The KS test gives a probability of $17 \%$ that the two distributions are consistent.

Fig. 9, we show the age distribution of both the whole sample (open histogram) and the 703 confirmed members (hatched histogram) in the case of $R_{V}=3.1$ and Siess et al. (2000) evolutionary models. The KS test evaluates a probability of $17 \%$ that the two distributions are drawn from the same parent population and the spread $(\sigma \log (a g e)=0.40)$ obtained for the subsample does not differ significantly from the value $\sigma \log ($ age $)=0.43$ derived for the whole sample. 
For these reasons, we are confident that contamination does not contribute very much to the scatter observed in the age distribution of the ONC.

\section{Age spread}

The observed dispersion in age represents an upper limit to the real age spread, being a combination of true age spread and scatter due to observational uncertainties. These, however, cannot explain the scatter of $\sim 1$ dex observed in the HR diagram, as we have found in the previous sections. To quantify, or at least to place a constraint, on the real age spread it is necessary to run a second Monte Carlo simulation to account for observational errors.

To increase the reliability of our results, we limited our analysis to the mass range in which our completeness is above $80 \%$. Our first step has been to test the hypothesis of an exactly coeval cluster, simulating a sample of stars with luminosities and temperature corresponding to a 2.2 Myr isochrone (assuming the mean age for the ONC estimated in Sect. 5) according to the Siess et al. (2000) evolutionary models. We then varied their luminosities assuming a Gaussian distribution of errors with $\sigma(\log L)=0.10$ (see Sect. 4), recovered their ages by interpolating over the Siess et al. (2000) isochrones and evolutionary tracks, and studied the overall age distribution. The spread around the isochrone is $\sigma(\log t)=0.13$. We compared this distribution with the observed one using the KS test and found a probability of $10^{-19}$ that the two distributions are drawn from the same parent population. The comparison is shown in Fig. 10. This formally confirms that the observational uncertainties alone are unable to account for the age spread observed in the region.

We then performed other simulations to find the amount of age spread needed to reproduce the observations, in addition to the dispersion caused by observational uncertainties. In Fig. 11, we show the comparison with the simulations in the cases of spreads of $1,1.5,2,2.5,3,3.5$, and 4 Myr. The probabilities given by the KS test are also shown in each panel of Fig. 11. Since for spreads larger than 3.5 and smaller than 1.5 Myr the KS test probability drops below $1 \%$, we suggest that the real age spread is between 1.5 and $3.5 \mathrm{Myr}$.

Therefore, on the basis of the isochrones, we find a spread of roughly 2-3 Myr in age, which is in good agreement with the results presented by Da Rio et al. (2010a), and other techniques for inferring stellar ages also confirm these results (Muench et al. 2008). Another way interpreting this result is that we observe a scatter in stellar radii at any given mass. The same evidence was found by Jeffries (2007) using the rotation periods and projected equatorial velocities $(v \sin i)$ for a sample of stars in the ONC. Jeffries (2007) studied the distribution of $R \sin i$ and modeled it as a dispersion in the true radii, inferring a spread in stellar ages of approximately a few Myr.

With reference to the recent attempts, Baraffe et al. (2009), Jeffries (2011), and Littlefair et al. (2011) with different methods suggest that the spread in radius observed in different starforming regions may be due to the accretion history and not to a real spread in age. Baraffe et al. (2009) present a theoretical calculation for the expected scatter in luminosity due to episodic accretion, while Jeffries (2011) find that stars with different ages do not show differences in accretion and disk excess and propose they have the same age. Finally, Littlefair et al. (2011) observe a correlation between rotation velocity and luminosity, which is the opposite of that expected in the case of a real age spread. This, according to the authors, might support
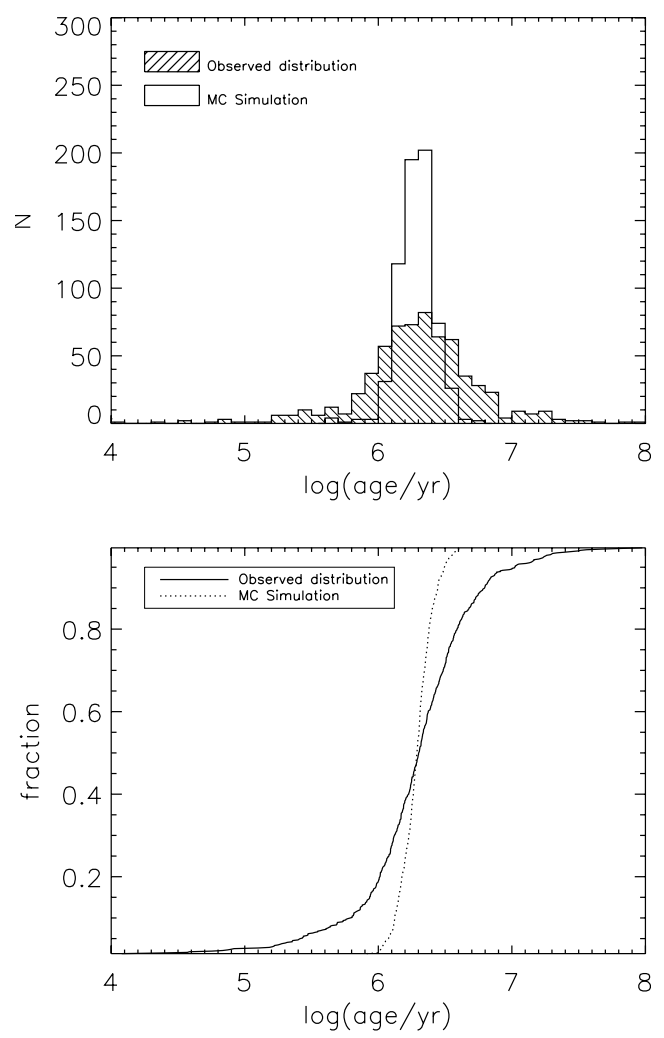

Fig. 10. Test of coeval population. Top: The open histogram represents the age distribution simulated for a sample of stars uniformly distributed along a 2.2 Myr isochrone from Siess et al. (2000), assuming an observational uncertainty in the luminosity of $\sigma(\log L)=0.10$. The spread in age is $\sigma(\log t)=0.13$. The hatched histogram shows the observed age distribution for the observed completeness-limited subsample. Bottom: Cumulative functions of the the two distributions. Solid and dotted line represent the observed and simulated sample, respectively.

of the scenario presented in Baraffe et al. (2009). In contrast, Hosokawa et al. (2011) show that the broadening of the PMS sequence in the HRD due to episodic accretion computed by Baraffe et al. (2009), was significantly overestimated, indicating that even this scenario might be incomplete.

At the moment, we cannot exclude that a combination of a real age spread and episodic accretion is required to explain the observations, especially since a model quantifying whether the accretion-induced luminosity spread is able alone to account for the observations has not yet been developed. In any case, a more complete scenario still needs to be investigated.

Finally, we note that the Orion nebula hosts a few sites of ongoing star formation, such as the BN/KL system, Ori-1 South, and an extremely dense filament of gas and dust stretching through the center of the ONC, which hosts at least 45 protostellar candidates (Muench et al. 2008). In our study of the optically visible population, we have not taken into account these embedded regions. Our finding that the mean age of the ONC is $2 \mathrm{Myr}$ and that the star formation appears to have occurred 1-3 Myr ago is consistent with the currently detected level of ongoing star formation. However, if future observations reveal dozens of more deeply embedded protostars over the same field of view as we observe several hundreds of $\mathrm{T}$ Tauri stars with ages of 1-3 Myr, the overall picture might change. 

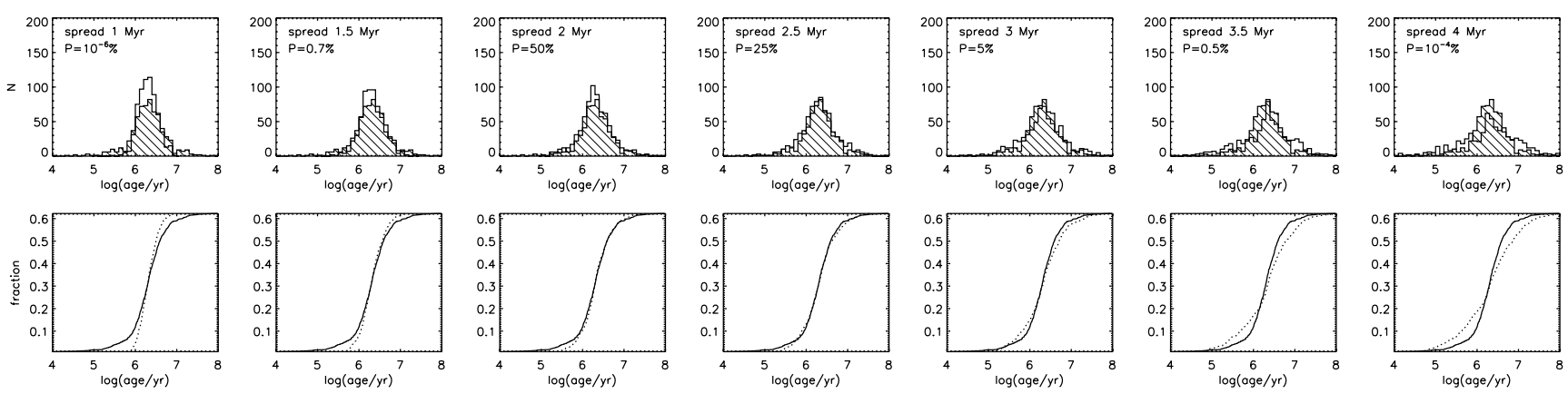

Fig. 11. Age spread test. Top, from left to right: the open histograms represent the age distribution simulated for a sample of stars of mean age of 2.2 Myr, assuming an observational uncertainty on the luminosity of $\sigma(\log L)=0.10$ and intrinsic age spreads of $1,1.5,2,1.5,3,1.5$, and $4 \mathrm{Myr}$, respectively. The KS test probability that the two distributions are drawn from the same parent function is also given. Bottom: cumulative functions for each simulation. We use the same legend as in Fig. 10.

\section{Spatial age distribution}

In Sect. 5, we derived the age distribution for the ONC according to different evolutionary models and reddening laws. We discuss now whether this distribution holds true at different locations in the cluster.

We limit again our investigation of the cluster age structure to the subsample of stars for which our completeness is above $80 \%$. In Fig. 12, we show the spatial distribution on the sky of this subsample of ONC stars, binned by age. Each bin has roughly the same number of stars. As can be easily seen, the youngest stars are more clustered towards the center, while the oldest ones are distributed almost homogeneously in space. This evidence of an age segregation was already noticed by Hillenbrand (1997). A two dimensional KS test also confirms this first-look impression. According to the KS test, when comparing the spatial distributions of stars younger than the mean age of the cluster (first two panels) we get a probability of $16 \%$ that they are consistent with each other, whereas we find a probability $<1 \%$ that they agree with the spatial distributions of the stars in the last two bins. Moreover, more than $65 \%$ of the stars younger than $1 \mathrm{Myr}$ are located within one parsec from the central $\mathrm{O}$ star, $\theta^{1}$ Ori $\mathrm{C}$, which is commonly assumed as center of the cluster.

This preliminary result suggests that there is a slight dependence of age on spatial distribution. However, a part of this trend might be biased by spatially variable incompleteness. While our completeness function is representative, on average, of the whole stellar sample that we have considered, the completeness varies with position, being lower toward the center of the system where the sky background is brighter. If this "age segregation" were confirmed, it might have implications for the study of the dynamical evolution of the cluster.

\section{Dynamical evolution of the ONC}

There have been many attempts to understand the dynamical history of the ONC (e.g. Hillenbrand \& Hartmann 1998; Allison et al. 2009). In particular, the evidence of mass segregation (Hillenbrand 1997; Hillenbrand \& Hartmann 1998) in such a young cluster highlights the problem of distinguishing between primordial and dynamical mass segregation. The cluster crossing time is $\sim 2 \mathrm{Myr}$, similar to the mean age of the cluster, and an even longer time is required for the cluster to relax (Hillenbrand \& Hartmann 1998). Thus, mass segregation might be primordial, meaning that the ONC formed with the most massive stars concentrated near the center. However, simulations of initially cool and clumpy clusters have shown that with these initial conditions mass segregation could also be dynamical on very short timescales (Allison et al. 2009). If the observed spread in radii were interpreted as a true age spread and the star formation in the ONC lasted for at least $2 \mathrm{Myr}$, the dynamics of the youngest and oldest stars might have been very different: the evolution of the oldest, earliest stars might have been dominated by the molecular cloud in which they were embedded, whereas the youngest stars in the cluster might have been affected by the dynamical influence of the stars that had already formed. This highlights that models that attempt to explain dynamical evolution in this region are probably still incomplete.

\section{Conclusions}

Taking advantage of the broad-band photometry extracted from the large data-set of ACS/WFC observations collected for the HST Treasury Program on the ONC, we have derived the HR diagram of the cluster, with the main goal of studying the age distribution and age spread in the region.

Our method is based on the estimate of the extinction and accretion luminosity towards each source by performing synthetic photometry on an empirical calibration of atmospheric models (Da Rio et al. 2010a) using the package Chorizos (Maíz-Apellániz 2004). This tool has allowed us to derive an accurate estimate of the intrinsic luminosity of each star. Taking into account the uncertainties in the distance, spectral type, extinction, unresolved binaries, accretion, and photometric variability, we have been able to determine both luminosity and age with $\log$ arithmic errors of $\sigma(\log l)=0.10$ and $\sigma(\log t)=0.15$, respectively. Compared to Hartmann (2001), we derived errors that are small enough to preserve a time dependence of the star formation rate possibly present in the observations, which is instead lost when the observational errors are $\sigma(\log t) \gtrsim 0.2$.

The HR diagram we derived shows a large spread in luminosity. According to the evolutionary calculations of Siess et al. (2000), the mean age of the cluster is $2.2 \mathrm{Myr}$, and is slightly dependent on the assumed reddening law. The dependence on the evolutionary models is much stronger since the choice of different isochrones and tracks affects both the mean age of the cluster and the estimate of the age of the single star. There are currently several evolutionary models available and our ability of indicating the best one is limited to secondary clues (e.g. spacial distribution and kinematics vs. average cluster age) and our prejudice (for instance lack of mass-age relation). However, the presence of an observed spread larger than the errors in the age is common to all the models. 
M. Reggiani et al.: Age spread in the ONC
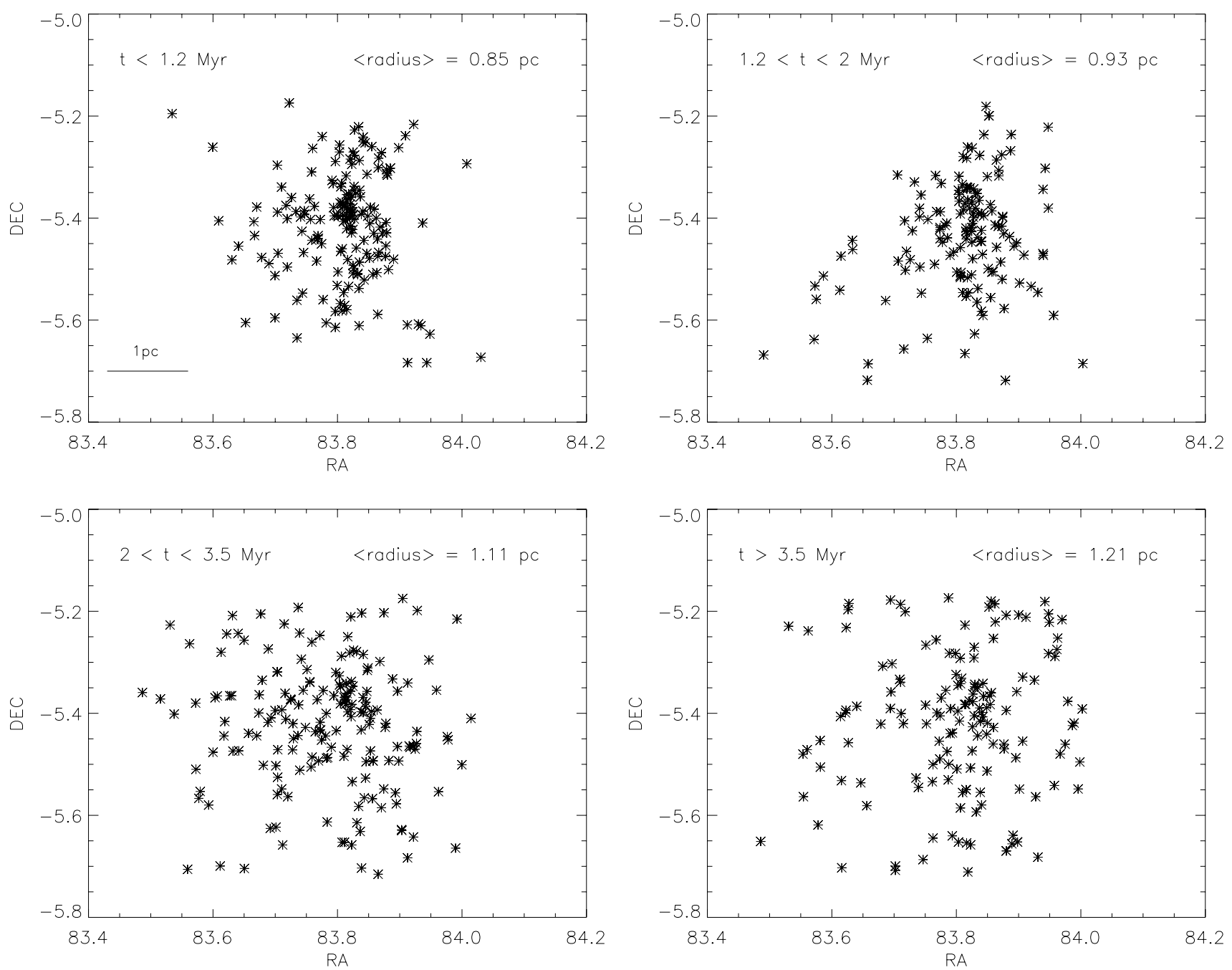

Fig. 12. Spatial age distribution. The four images show the spatial distribution of stars within various age ranges. The youngest stars are clearly more centered towards the center than the oldest ones.

A preliminary study in this paper of the spatial distribution of the ages suggests that there is age segregation in the ONC. We cannot exclude that such a segregation is caused by the spatial dependence of the completeness, although further investigation is certainly needed.

To quantify the amount of intrinsic luminosity spread in terms of age spread, we have also performed MC simulations taking into account the sources of errors mentioned before. First of all, the observations are inconsistent with a coeval stellar population, even if we account for the apparent age spread due to observational uncertainties. Second, the observed age distribution in the ONC is consistent with simulations of stellar population with an intrinsic age spread between 1.5 and 3.5 Myr, but not larger. These results confirm the presence of a real luminosity spread, which cannot be accounted for entirely by observational uncertainties but reflects a real spread in the stellar radii for a given mass. We have interpreted this as a true age spread in Orion, implying that star formation in this region lasted a couple of Myr. Some authors suggest that this spread might be due to the accretion history but at the moment the truthfulness of this scenario has not yet been proven. However, any possible explanation for the spread observed in the HR diagram should reproduce this amount of age spread. Within the current limitations, we conclude that the age is $2 \mathrm{Myr}$ and the age spread is $\sim 2 \mathrm{Myr}$.
Acknowledgements. We thank an anonymous referee for valuable suggestions. Support for program 10246 was provided by NASA through a grant from the Space Telescope Science Institute (STScI), which is operated by the Association of Universities for Research in Astronomy, Inc., under NASA contract NAS 526555. A portion of the work of M. Reggiani was done under the auspices of the Summer Student Program of the Space Telescope Science Institute. L.R. acknowledges the Ph.D. fellowship of the International Max-Planck-Research School.

\section{References}

Allard, F., Hauschildt, P. H., \& Schwenke, D. 2000, ApJ, 540, 1005 Allison, R. J., Goodwin, S. P., Parker, R. J., et al. 2009, ApJ, 700, L99 Baraffe, I., Chabrier, G., Allard, F., \& Hauschildt, P. H. 1998a, A\&A, 337, 403 Baraffe, I., Chabrier, G., Allard, F., \& Hauschildt, P. 1998b, VizieR Online Data Catalog, 333, 70403

Baraffe, I., Chabrier, G., \& Gallardo, J. 2009, ApJ, 702, L27

Berdyugina, S. V. 2005, Liv. Rev. Sol. Phys., 2, 8

Binney, J., \& Merrifield, M. 1998, Galactic astronomy/James Binney and Michael Merrifield (Princeton, NJ: Princeton University Press), Princeton series in astrophysics

Brown, A. G. A., de Geus, E. J., \& de Zeeuw, P. T. 1994, A\&A, 289, 1

Calvet, N., \& Gullbring, E. 1998, ApJ, 509, 802

Costero, R., \& Peimbert, M. 1970, Boletin de los Observatorios Tonantzintla y Tacubaya, 5, 229

D’Antona, F., \& Mazzitelli, I. 1994, ApJS, 90, 467

D’Antona, F., \& Mazzitelli, I. 1997, Mem. Soc. Astron. Ital., 68, 807

Da Rio, N., Robberto, M., Soderblom, D. R., et al. 2009, ApJS, 183, 261

Da Rio, N., Robberto, M., Soderblom, D. R., et al. 2010a, ApJ, 722, 1092 
Da Rio, N., Gouliermis, D. A., \& Gennaro, M. 2010b, ApJ, 723, 166

Duquennoy, A., \& Mayor, M. 1991, A\&A, 248, 485

Ferland, G. J., Korista, K. T., Verner, D. A., et al. 1998, PASP, 110, 761

Hartmann, L. 2001, AJ, 121, 1030

Hartmann, L. 2009, Accretion Processes in Star Formation, second edition, ed.

L. Hartmann (Cambridge, UK: Cambridge University Press)

Hauschildt, P. H., Allard, F., Ferguson, J., Baron, E., \& Alexander, D. R. 1999, ApJ, 525, 871

Herbst, W., Bailer-Jones, C. A. L., Mundt, R., Meisenheimer, K., \& Wackermann, R. 2002, A\&A, 396, 513

Hillenbrand, L. A. 1997, AJ, 113, 1733

Hillenbrand, L. A., \& Hartmann, L. W. 1998, ApJ, 492, 540

Hillenbrand, L. A., \& Carpenter, J. M. 2000, ApJ, 540, 236

Hillenbrand, L. A., \& White, R. J. 2004, ApJ, 604, 741

Hillenbrand, L. A., Bauermeister, A., \& White, R. J. 2008, 14th Cambridge

Workshop on Cool Stars, Stellar Systems, and the Sun, 384, 200

Hosokawa, T., Offner, S. S. R., \& Krumholz, M. R. 2011, ApJ, 738, 140

Johnson, H. L. 1967, ApJ, 150, L39

Jones, B. F., \& Walker, M. F. 1988, AJ, 95, 1755

Kurucz, R. L. 1993, VizieR Online Data Catalog, 6039, 0

Jeffries, R. D. 2007, MNRAS, 381, 1169

Jeffries, R. D. 2011 [arXiv: 1102.4752]

Littlefair, S. P., Naylor, T., Mayne, N. J., Saunders, E., \& Jeffries, R. D. 2011 [arXiv: 1102.3836]

Luhman, K. L. 1999, ApJ, 525, 466
Maíz-Apellániz, J. 2004, PASP, 116, 859

McNamara, B. J., Hack, W. J., Olson, R. W., \& Mathieu, R. D. 1989, AJ, 97, 1427

Menten, K. M., Reid, M. J., Forbrich, J., \& Brunthaler, A. 2007, A\&A, 474, 515

Muench, A. A., Lada, E. A., Lada, C. J., \& Alves, J. 2002, ApJ, 573, 366

Muench, A., Getman, K., Hillenbrand, L., \& Preibisch, T. 2008, Handbook of Star Forming Regions, I, 483

Palla, F., \& Stahler, S. W. 1999, ApJ, 525, 772

Palla, F., Randich, S., Flaccomio, E., \& Pallavicini, R. 2005, ApJ, 626, L49

Palla, F., Randich, S., Pavlenko, Y. V., Flaccomio, E., \& Pallavicini, R. 2007, ApJ, 659, L41

Press, W. H., Teukolsky, S. A., Vetterling, W. T., \& Flannery, B. P. 1992 (Cambridge: University Press), 2nd ed.

Reipurth, B., Guimarães, M. M., Connelley, M. S., \& Bally, J. 2007, AJ, 134, 2272

Ricci, L., Robberto, M., \& Soderblom, D. R. 2008, AJ, 136, 2136

Robberto, M., Soderblom, D. R., Scandariato, G., et al. 2010, AJ, 139, 950

Siess, L., Dufour, E., \& Forestini, M. 2000, A\&A, 358, 593

Slesnick, C. L., Hillenbrand, L. A., \& Carpenter, J. M. 2004, ApJ, 610, 1045

Stassun, K. G., Mathieu, R. D., Mazeh, T., \& Vrba, F. J. 1999, AJ, 117, 2941

van Altena, W. F., Lee, J. T., Lee, J.-F., Lu, P. K., \& Upgren, A. R. 1988, AJ, 95, 1744

Walker, M. F., \& Jones, B. F. 1988, PASP, 100, 1505

Weights, D. J., Lucas, P. W., Roche, P. F., Pinfield, D. J., \& Riddick, F. 2009, MNRAS, 392, 817 